\title{
The orientation and current density of the magnetotail current sheet: A statistical study of the effect of geomagnetic conditions
}

\author{
E. A. Davey, ${ }^{1}$ M. Lester, ${ }^{1}$ S. E. Milan, ${ }^{1}$ R. C. Fear, ${ }^{1}$ and C. Forsyth ${ }^{2}$
}

Received 12 March 2012; revised 7 June 2012; accepted 9 June 2012; published 24 July 2012.

[1] We examine the orientation and current density of the current sheet during current sheet crossings from Cluster's 2001-2007 tail seasons. The curlometer technique is used to estimate the current density and is combined with Minimum Variance Analysis (MVA) to calculate the direction of the current sheet normal. The SYM-H and AE indices at the time of each crossing are employed to assess how the tilt angle (the angle the normal makes with the $\mathrm{Z}$ axis in the GSM YZ plane) and current density depend on geomagnetic conditions. Our results indicate a larger current sheet tilt in the $\mathrm{YZ}$ plane during intervals of stronger and/or more prolonged substorm activity, as indicated by the AE index. There is also evidence that when the ring current is enhanced during magnetic storms, the current sheet is less tilted even though the AE index is also disturbed. In addition larger current densities are seen during times of both magnetic storms and substorms, compared to crossings during only substorms and a quiet ring current. We conclude that increased substorm activity disrupts the current sheet structure resulting in greater motion of the current sheet (as found by Davey et al. (2012)) and a greater local tilt to the current sheet. We propose that the increased open flux in the tail during magnetic storms stabilizes the current sheet such that the tilt angle of the current sheet is reduced. The increased amount of open flux during magnetic storms also results in larger current densities within the current sheet.

Citation: Davey, E. A., M. Lester, S. E. Milan, R. C. Fear, and C. Forsyth (2012), The orientation and current density of the magnetotail current sheet: A statistical study of the effect of geomagnetic conditions, J. Geophys. Res., 117, A07217, doi:10.1029/ 2012JA017715.

\section{Introduction}

[2] The cross-tail current sheet is one of the key regions in substorm and storm processes. It separates the northern and southern lobes of oppositely directed magnetic field and is located in the center of the plasma sheet. The structure and dynamics of the cross-tail current sheet have been widely studied and the Cluster mission has enabled far greater understanding of its complex nature by exploiting multispacecraft techniques than previously possible from singlespacecraft missions. Spacecraft have been observed to make multiple crossings of the current sheet during a single orbit, evidenced by multiple reversals of the $\mathrm{B}_{X}$ component of the magnetic field, indicating that the current sheet is undergoing a flapping motion. The exact processes which cause this current sheet motion are as yet undetermined, but this motion has previously been associated with substorms [e.g., Davey

\footnotetext{
${ }^{1}$ Department of Physics and Astronomy, University of Leicester, Leicester, UK.

${ }^{2}$ Mullard Space Science Laboratory, University College London, London, UK.

Corresponding author: E. A. Davey, Department of Physics and Astronomy, University of Leicester, University Road, Leicester LE1 7RH, UK. (ead13@ion.le.ac.uk)

(C)2012. American Geophysical Union. All Rights Reserved. 0148-0227/12/2012JA017715
}

et al., 2012], solar wind influences [Forsyth et al., 2009] and bursty bulk Flows (BBFs) [e.g., Sergeev et al., 2006].

[3] Studies have shown that when the current sheet is active (during flapping motion), it can be highly tilted in the YZ GSM plane and that this tilt is reduced during periods of inactivity when the current sheet is not undergoing flapping motion. For example, Zhang et al. [2002] calculated the inclination angle of the current sheet in the YZ GSM plane using 4 point timing methods for five crossings in 2001 of an active current sheet by Cluster and found that this angle ranged from $41^{\circ}$ to over $90^{\circ}$. A further study by Sergeev et al. [2003] found large tilt angles of $60-70^{\circ}$ in the $\mathrm{YZ}$ plane using inter-spacecraft timing and MVA methods. A highly tilted current sheet during flapping motion was also shown by Sergeev et al. [2004] and Runov et al. [2005a], where the $\mathrm{Z}$ component of the current density measurements was larger than the Y component. In contrast, a statistical study of inactive current sheets during Cluster's 2001-2003 tail seasons by Petrukovich et al. [2005] showed lower tilt angles from 0 to $40^{\circ}$. They also found that the tilt of the current sheet increased toward the flanks of the magnetotail. While these studies have shown that the current sheet tilt is highly variable, there has been no systematic study of which factors stabilize the variation. We will address this issue in this paper.

[4] Observations have shown that the current density in the tail current sheet can range from less than $10 \mathrm{nA} \mathrm{m}^{-2}$ 
[Shen et al., 2008; Sergeev et al., 2003] to values as high as $30 \mathrm{nA} \mathrm{m}^{-2}$ [Runov et al., 2005a, 2006]. Values estimated using high spacecraft separation may be underestimated due to the linear interpolation between the spacecraft nodes. However, Nakamura et al. [2006] noted current density values of the order of $100 \mathrm{nA} \mathrm{m}^{-2}$ during a substorm interval, using a $200 \mathrm{~km}$ Cluster separation. Comparing these studies indicates that the current density in the current sheet tends to be larger when the sheet is active, but as yet this has not been shown statistically nor has any physical mechanism been proposed to explain this. We will also study these questions in this paper.

[5] Using data from the 2001-2007 Cluster tail seasons, we investigate the dependence of the orientation and current density of the tail current sheet on geomagnetic activity and location. We use the SYM-H and AE indices as measures of geomagnetic activity at the time of each crossing of the current sheet. We examine whether or not the tail is more inclined toward the flanks and whether or not the tail is more highly inclined during periods of greater geomagnetic activity. We also investigate the current density within the sheet and how it varies with geomagnetic activity. We discuss our findings in the context of the global configuration of the magnetosphere and how this configuration may impede activity in the magnetotail.

\section{Instrumentation}

[6] We have employed data from the Cluster Fluxgate Magnetometers from Cluster 1-4 (FGM) [Balogh et al., 2001; Gloag et al., 2010] and the Cluster Ion Spectrometer CODIF sensor (CIS-CODIF) [Rème et al., 2001; Dandouras et al., 2010] from Cluster 3. The FGM data are the $5 \mathrm{~Hz}$ resolution data from the Cluster Active Archive (CAA) [Perry et al., 2006; Laakso et al., 2010], box-car averaged and re-binned to a 1 second resolution. The FGM and CIS instrument data were used to search for current sheet crossings as well as to calculate the current density and tilt angle. We have also employed SYM-H [Iyemori, 1990] and AE [Davis and Sugiura, 1966] data from the WDC for Geomagnetism, Kyoto, to provide information on the level of magnetic activity at the time of each current sheet crossing.

\section{Current Sheet Crossing Identification and Data Analysis Techniques}

\subsection{Selection of Data Set}

[7] In a recent study, Davey et al. [2012] identified the number of current sheet crossings per orbit for Cluster 3 in the 2001-2007 tail seasons. Orbits in which there were large data gaps $(>480 \mathrm{~s})$ and in which the $\mathrm{H}^{+}$density was greater than $1.5 \mathrm{~cm}^{-3}$ were excluded to avoid missing any crossings or including crossings in the magnetosheath. Davey et al. [2012] investigated various thresholds in $\mathrm{B}_{X}( \pm 3 \mathrm{nT}, \pm 5$ $\mathrm{nT}$ and $\pm 7 \mathrm{nT}$ ) to define a crossing and no significant differences were found in the results, between the different thresholds. Consequently, Davey et al. [2012] used the $\pm 5 \mathrm{nT}$ threshold for a change in $\mathrm{B}_{X}$ to define a crossing when the spacecraft was located at $\mathrm{X}<-8 R_{E}$ and $|Y|<10 R_{E}$. In this study, we use a similar set of criteria to Davey et al. [2012] to identify those orbits with large data gaps or in which the spacecraft passed into the magnetosheath. However, in this study we identify the current sheet crossings using the barycentric magnetic field data from the Cluster spacecraft, estimated to be the average magnetic field across all four spacecraft.

[8] Figure 1 shows the location of Cluster 3 during those parts of its orbit in which current sheet crossings were observed within the range $\mathrm{X}<-8 R_{E}$ and $|Y|<10 R_{E}$ (taken from Davey et al. [2012]). The data are shown in the $\mathrm{XZ}$ (Figure 1a), YZ (Figure 1b) and XY (Figure 1c) planes in GSM coordinates. The limits of the range of positions in terms of $\mathrm{X}$ and $\mathrm{Y}$ directions are due to the criteria used in the selection of crossings. The crossings occur in a range in $\mathrm{Z}$ of about -4 to $+5 \mathrm{R}_{E}$. The average tilt of the current sheet in the YZ plane has also been observed by Zhang et al. [2006] and Rong et al. [2010] and is discussed in Davey et al. [2012]. The analysis of the tilt angle of the current sheet, described in the next sections, shows the angles calculated for each crossing. The average tilt shown in Figure 1 in the $\mathrm{YZ}$ plane has not been subtracted from any of the following results, but we note that it is of the order of $13^{\circ}$.

\subsection{Data Analysis Methods}

[9] For each crossing, the barycentric magnetic field (that is the magnetic field at the center of mass of the spacecraft tetrahedron) was estimated as the mean magnetic field across the four spacecraft. Only those crossings which involved a current sheet crossing within the barycentric data were used for the study.

[10] Minimum variance analysis (MVA) [Sonnerup and Scheible, 1998] was used to identify the direction of maximum variance (I) in the barycentric magnetic field data for each crossing. All crossings included in the data set had a ratio of maximum to intermediate variance eigenvalues of greater than 4. The curlometer technique [Dunlop et al., 1988; Robert et al., 1998] was used to estimate the magnitude and direction ( $\mathbf{m})$ of the current density throughout the crossing. We performed a cross product of the current density direction (m) and the maximum variance direction (l) to estimate the direction normal to the current sheet (n). The angle that the normal direction makes with the $\mathrm{Z}$ (GSM) axis $\left(\arctan \left(\left|\mathrm{n}_{Y}\right| /\left|\mathrm{n}_{Z}\right|\right)\right)$ was defined as the tilt angle of the current sheet during each crossing. The effective position from the center of the current sheet, or vertical scale along the normal $\left(Z^{*}\right)$ was calculated using

$$
Z^{*}(t)=\int_{t_{1}}^{t_{2}} \frac{\partial B_{l}}{\partial t}\left[\nabla_{n} B_{l}\right]^{-1} \mathrm{~d} t
$$

taken from Runov et al. [2005b], where $\nabla_{n}$ is the gradient in the normal direction of the current sheet and $\mathrm{B}_{l}$ is the component of the magnetic field in the direction of maximum variance. When the current density is plotted as a function of $Z^{*}$ it provides a profile of the current density and the half thickness of the current sheet can be estimated.

[11] The curlometer technique assumes that the magnetic field varies linearly between the spacecraft. It estimates the current density within the tetrahedron and is a combination of the estimates of the current density through the faces of the spacecraft tetrahedron. In the calculations, the divergence of the magnetic field deviates from Gauss's Law, from 

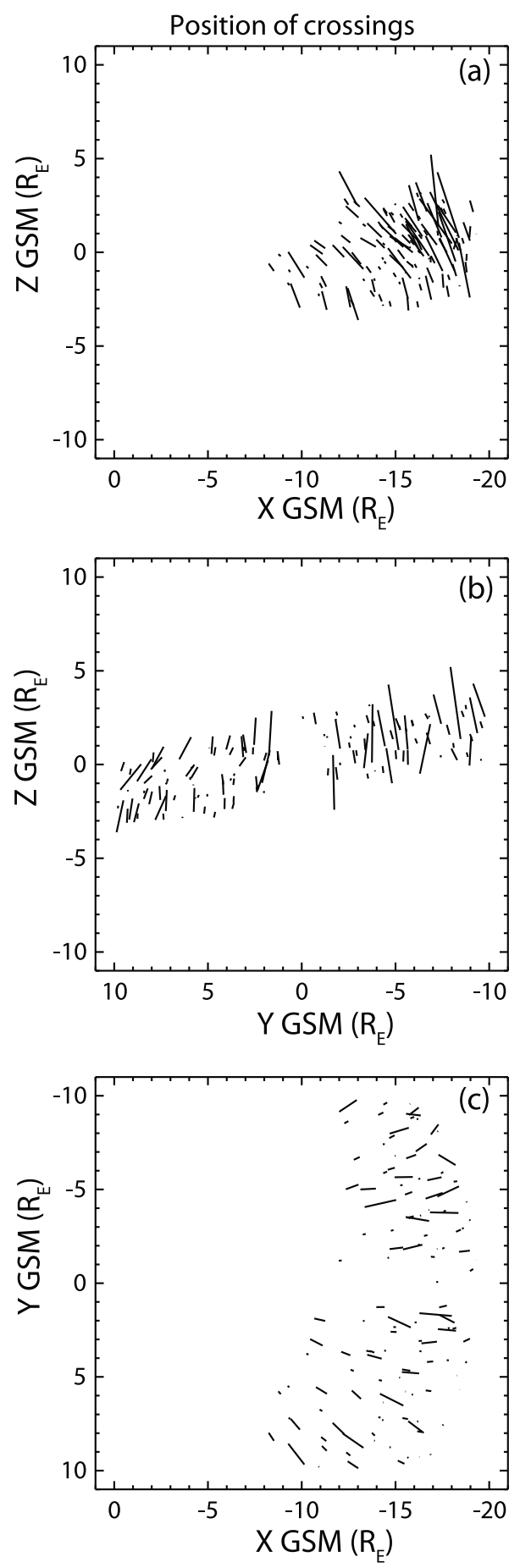

Figure 1. Position of the orbits containing the current sheet crossings for each pass through the magnetotail from Davey et al. [2012]. Each part orbit that contains all current sheet crossings is indicated by a line, shown in the (a) XZ, (b) YZ and (c) XY GSM planes. The crossings are defined as a change in $\mathrm{B}_{X}$ from $5 \mathrm{nT}$ to $-5 \mathrm{nT}$ or vice versa.

errors in the data and the assumptions used in the curlometer technique, and is used to provide a measure of the accuracy of the estimates. Crossings were included if the accuracy of the current density estimate, indicated by the ratio of div B to curl B, was less than 0.3 for more than $60 \%$ of the crossing, based on the work by Runov et al. [2005b]. The database of crossings used in the present study comprised 279 crossings. For each crossing within the database, the SYM-H and AE indices at the time of the crossings i.e. where the barycentric $\mathrm{B}_{X}=0$, were used to identify whether the ring current was enhanced and whether substorms were present.

\section{Results}

\subsection{Current Sheet Orientation}

[12] Figure 2 shows how the current sheet tilt angle varies with the SYM-H (Figure 2a) and AE (Figure 2b) indices at the time of each crossing. When SYM-H is $<-70 \mathrm{nT}$ (in Figure 2a), the tilt angles are $<25^{\circ}$. In addition there are very high values of tilt angle, approaching $90^{\circ}$, that occur when SYM-H is positive and also when it is negative down to about $-50 \mathrm{nT}$. In addition, the scatter of values increases as SYM-H increases (up to about $+20 \mathrm{nT}$ ). The variation of tilt angle with the AE index is however not so clear (Figure 2b). For the purposes of this paper, we have used the SYM-H index to give an indication of when the ring current is enhanced and therefore we define storm conditions to be when SYM-H values are $\leq-50 \mathrm{nT}$. In view of this, the tilt angle variation with the $\mathrm{AE}$ index for non-storm times (where SYM-H $>-50 \mathrm{nT}$ ) is indicated by the black data points and storm-times (where SYM-H $\leq-50 \mathrm{nT}$ ) by the red data points. For large AE values ( $>500 \mathrm{nT})$ the non-storm times (black data points) have, in general, higher tilt angles than the storm-time data (red data points), although there are fewer data in this region compared to where $\mathrm{AE}<500 \mathrm{nT}$. For lower values of the $\mathrm{AE}$ index, there is a wide range of tilt angles, with most crossings having tilt angles of less than $25^{\circ}$. However, there are also some crossings with tilt angles of $>60^{\circ}$ when the $\mathrm{AE}$ index is low and in general there is no obvious trend for $\mathrm{AE}$ values $<500 \mathrm{nT}$. In order to view the distributions more clearly, Figure 3 shows the mean tilt angle in bins of SYM-H (Figure 3a) and AE (Figure 3b). As in Figure 2, Figure 3b shows non-storm time data in black with storm data in red. Vertical lines through each bar indicate the standard errors on the means and the numbers in each bin are shown above each bar. There is a peak in mean tilt angle where SYM-H is between 0 and $50 \mathrm{nT}$ in Figure $3 \mathrm{a}$, with a decrease in tilt angle as the bins approach $-150 \mathrm{nT}$. In Figure $3 b$ the mean tilt angle increases with higher values of the AE index, although the number of data points decreases above $500 \mathrm{nT}$. To investigate the significance of the relationship with the AE index, we applied the Mann-WhitneyWilcoxon test [Barlow, 1989]. The test is used to compare groups when the distribution cannot be assumed to be normal. It tests whether the two groups are from the same population and uses a ranking system on the data, resulting in a $U$ statistic that can be compared to tables of critical values. When comparing the tilt angles in a group with low $\mathrm{AE}$ indices $(<500 \mathrm{nT})$ compared to a group with high AE indices $(\geq 500 \mathrm{nT})$, for non-storm times only (SYM-H $>-50 \mathrm{nT}$ ), the mean tilt angle for the lower $\mathrm{AE}$ group is $16.95^{\circ}$ and $27.60^{\circ}$ for the high AE group. The Mann-Whitney-Wilcoxon test demonstrates that the two groups are statistically different at the $95 \%$ level.

[13] In order to see any trends in tilt angle with position, the mean tilt angle of the current sheet for groups of crossings in $1 \mathrm{R}_{E}$ bins, at different positions in the magnetotail is 

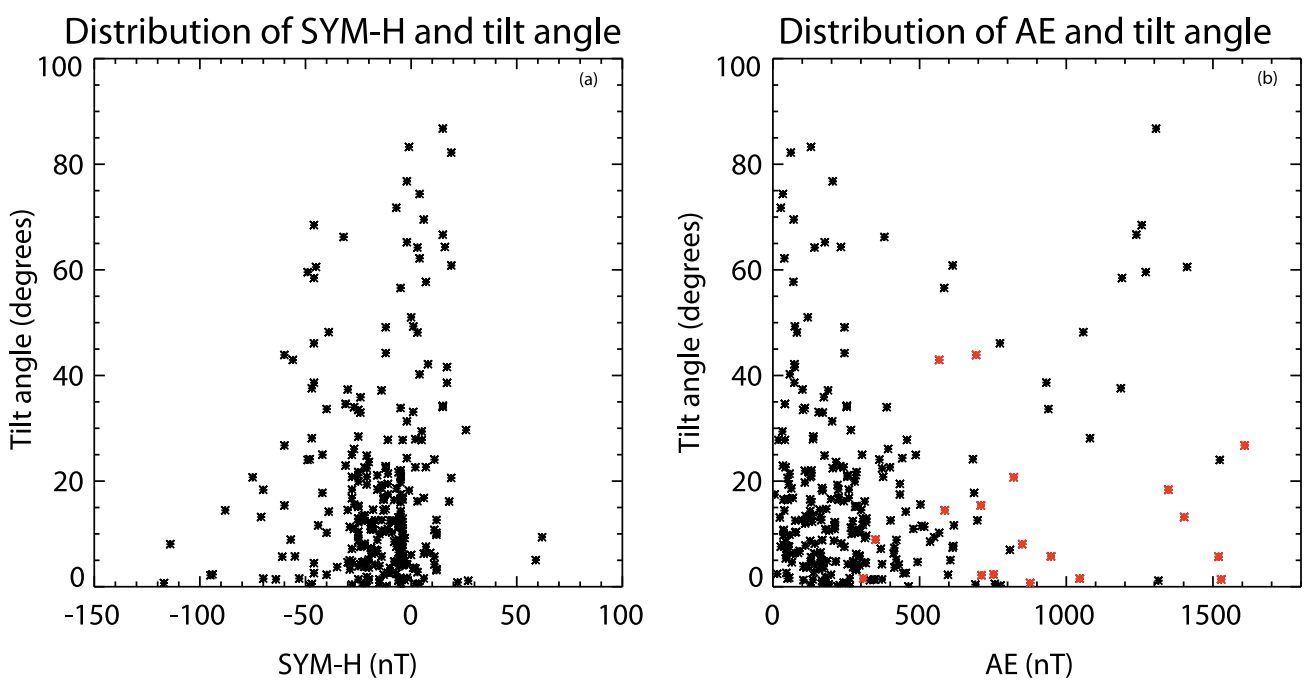

Figure 2. Distributions of tilt angle for each crossing, with (a) SYM-H and (b) the AE index. In Figure 2b, black data points represent those crossings where SYM-H $>-50 \mathrm{nT}$ and the red data points are where SYM-H $\leq-50 \mathrm{nT}$. The values of SYM-H and the AE index used are those at the point of the crossing where $\mathrm{B}_{X}=0$.

plotted in black in Figure 4. The positions used are the start of each crossing in X (Figure 4a), Y (Figure 4b) and Z (Figure 4c) in GSM coordinates. Standard errors on the mean values are indicated by vertical lines through each data point. The numbers within each bin are indicated by the red bars (scale on the right hand side). The variation in the tilt angle with position in the $\mathrm{X}$ direction does not show any significant variation (Figure $4 a$ ). Some of the bins contain only small numbers of crossings, particularly at the extremes in X and Z. Larger tilt angles are evident in the dawn flank of the tail compared to those around $\mathrm{Y}=0$ (Figure $4 \mathrm{~b}$ ). There is no evidence that there are increased tilt angles on the dusk flank of the tail, although we note that our data are restricted to $|Y|<10 \mathrm{R}_{E}$, so we are unable to ascertain whether the tilt angles increase further into the dusk flank. Figure $4 \mathrm{c}$ shows larger tilt angles at larger $\mathrm{Z}$ values.

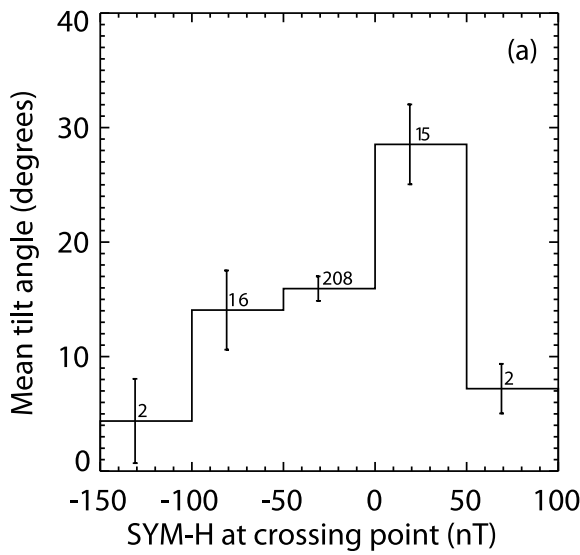

[14] Figure 5 shows the mean tilt angle for three different levels of geomagnetic activity i.e. during quiet times, substorms and magnetic storms (indicated by an enhanced ring current). The data were separated according to the $\mathrm{AE}$ and SYM-H indices at the time of each crossing. Quiet conditions (QT) were defined as SYM-H $>-50 \mathrm{nT}$ and $\mathrm{AE}<$ $500 \mathrm{nT}$. Substorm conditions (AE) were defined as SYM-H $>$ $-50 \mathrm{nT}$ and $\mathrm{AE} \geq 500 \mathrm{nT}$ and finally storm conditions with an enhanced ring current $(\mathrm{RC})$ were defined as SYM-H $\leq$ $-50 \mathrm{nT}$ and $\mathrm{AE} \geq 500 \mathrm{nT}$. It should be noted that the two crossings that occurred during enhanced ring current conditions with low values of the $\mathrm{AE}$ are not included in the analyses of the three groups mentioned previously as our aim is to compare the effect of the ring current under similar $\mathrm{AE}$ conditions. Standard errors on the means are shown by vertical lines through each data point and the numbers in each

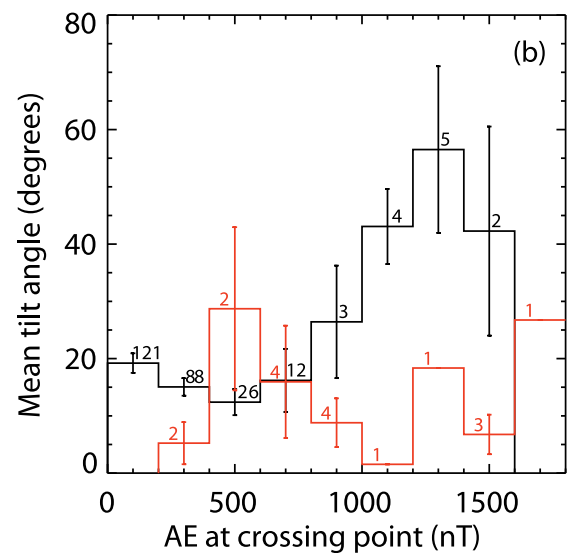

Figure 3. Distributions of mean tilt angle as a function of (a) SYM-H and (b) the AE index. Figure 3a shows the data in $50 \mathrm{nT}$ bins and Figure $3 \mathrm{~b}$ shows the data in $250 \mathrm{nT}$ bins. In Figure $3 \mathrm{~b}$, those crossings where SYM-Heater $-50 \mathrm{nT}$ are shown in black and SYM-H $\leq-50 \mathrm{nT}$ are the red data. The values of SYM-H and the AE index used are those at the point of the crossing where $\mathrm{B}_{X}=0$. Standard errors of the means are shown by vertical lines through each bar and the numbers in each bin are above each bar. 

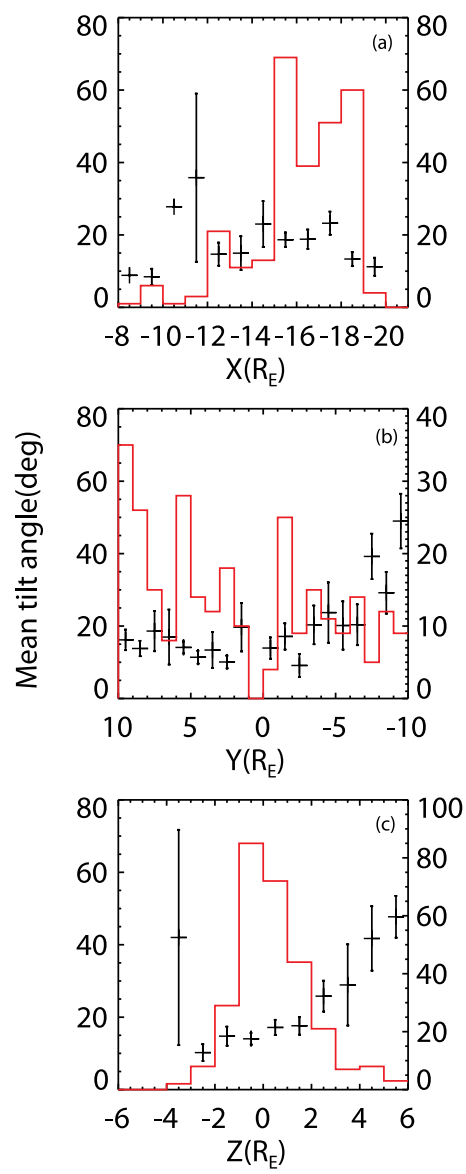

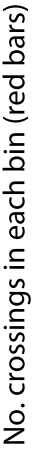

Figure 4. Mean tilt angle of the current sheet at the position of the start of the crossings, in terms of (a) X, (b) Y and (c) Z in GSM coordinates. Data is presented in $1 \mathrm{R}_{E}$ bins and the standard errors in the mean values are shown by vertical lines through each data point. The secondary $\mathrm{Y}$ axis shows the numbers in each bin group, indicated by the red bars.

group are shown above each bar. The tilt angle is largest for the substorm group (AE) whereas the enhanced ring current group (RC) has the lowest mean tilt angle. The Mann-Whitney-Wilcoxon test was again used to compare the $\mathrm{QT}$ and $\mathrm{AE}$ groups and the $\mathrm{AE}$ and $\mathrm{RC}$ groups. We found the differences between those groups to be statistically significant to a $95 \%$ significance level.

[15] Since the Cluster orbit changes from one year to the next we compare the tilt angle for different geomagnetic conditions for the first four years of data in Figure 6. The years 2005-2007 are not shown in this figure, as there are no crossings in the ' $\mathrm{RC}$ ' group, which would allow a comparison across all three levels of geomagnetic activity for these years. The classification of the different geomagnetic groups is the same as in Figure 5. Standard errors on the means are shown by vertical lines through each data point and the numbers in each group are shown above each bar. The mean values of SYM-H and the AE index, for each of the groups, are also shown next to each plot. Figures $6 \mathrm{a}-6 \mathrm{c}$ show that for 2001-2003, the 'AE' group has larger tilt angles compared to the ' $\mathrm{QT}$ ' and ' $\mathrm{RC}$ ' groups, although large variability is seen in the 'AE' group in 2002 and 2003. In 2004
(Figure 6d), the 'AE' group has a lower value of mean tilt angle compared to the other groups. The largest difference between the mean tilt angles of the 'AE' and ' $\mathrm{RC}$ ' groups is in 2001 (Figure 6a) where the mean SYM-H value for the 'RC' group is $-102 \mathrm{nT}$. In 2004, where the 'RC' group tilt angle is larger than the 'AE' group and the data do not follow the pattern of years 2001-2003, the mean SYM-H value for the ' $\mathrm{RC}$ ' group is $-59 \mathrm{nT}$, indicating a much less enhanced ring current than in the earlier years. Figure 6 indicates that there may be more of an effect of an enhanced ring current on the tilt angle when SYM-H is more negative as in 2001, compared to the later years when the values of SYM-H are less negative.

\subsection{Current Sheet Current Density}

[16] In this section, we analyze the current density in the magnetotail current sheet with respect to position and geomagnetic activity, in a similar manner to our treatment of the current sheet tilt angle in section 4.1. For each crossing, the maximum current density magnitude reached was calculated and Figure 7 shows the spatial distribution of the means of those maximum current density values in $1 \mathrm{R}_{E}$ bins in the $\mathrm{X}$ (Figure 7a), Y (Figure 7b) and Z GSM (Figure 7c) directions. As in Figure 4 some of the bins have smaller numbers of crossings and should be treated with caution. The mean current density magnitude shows no obvious trend with position, unlike the current sheet tilt angle (section 4.1).

[17] In Figure 8 the mean current density magnitude is plotted as a function of $Z^{*}$ in $0.1 \mathrm{R}_{E}$ bins, for each of the geomagnetic condition groups described earlier, using all the data from 2001-2007. The mean current density magnitude within each bin of $Z^{*}$ was calculated for each crossing and Figure 8 shows the means of those values for each geomagnetic activity group. The quiet conditions group (QT) is shown in red, the high AE index group (AE) is shown in green and the enhanced ring current group (RC) is in blue and they are defined in the same way as in previous figures. The standard errors on the mean values are indicated by vertical lines through each data point. When the data are considered together in this way, the ' $\mathrm{RC}$ ' and ' $\mathrm{QT}$ ' groups have larger maximum current densities than the 'AE' group, particularly in the central section of the current sheet, around $Z^{*}=0$.

[18] As with the tilt angle study (section 4.1), separating the data into the first four years of data enables a comparison of geomagnetic conditions within each tail season as well as an investigation of the effect of increasing strengths of the ring current. The mean current density data as a function of $\mathrm{Z}^{*}$, in $0.1 \mathrm{R}_{E}$ bins and separated by year, is presented (Figure 9) for the first four years of data where there are enough data to allow comparisons of all three geomagnetic condition groups. The mean values of SYM-H and AE are given for each group next to the panels. The geomagnetic conditions are indicated by the same colors as in the previous figure. Standard errors on the mean values are shown by vertical lines through each data point. Data from 2001 and 2002 show a larger maximum current density magnitude for the 'RC' group compared to the 'AE' and 'QT' groups. However, 2003 and 2004 data do not show the same results, with the 'RC' group having similar maximum current density values as the 'AE' group. It is interesting to note that for 2001 


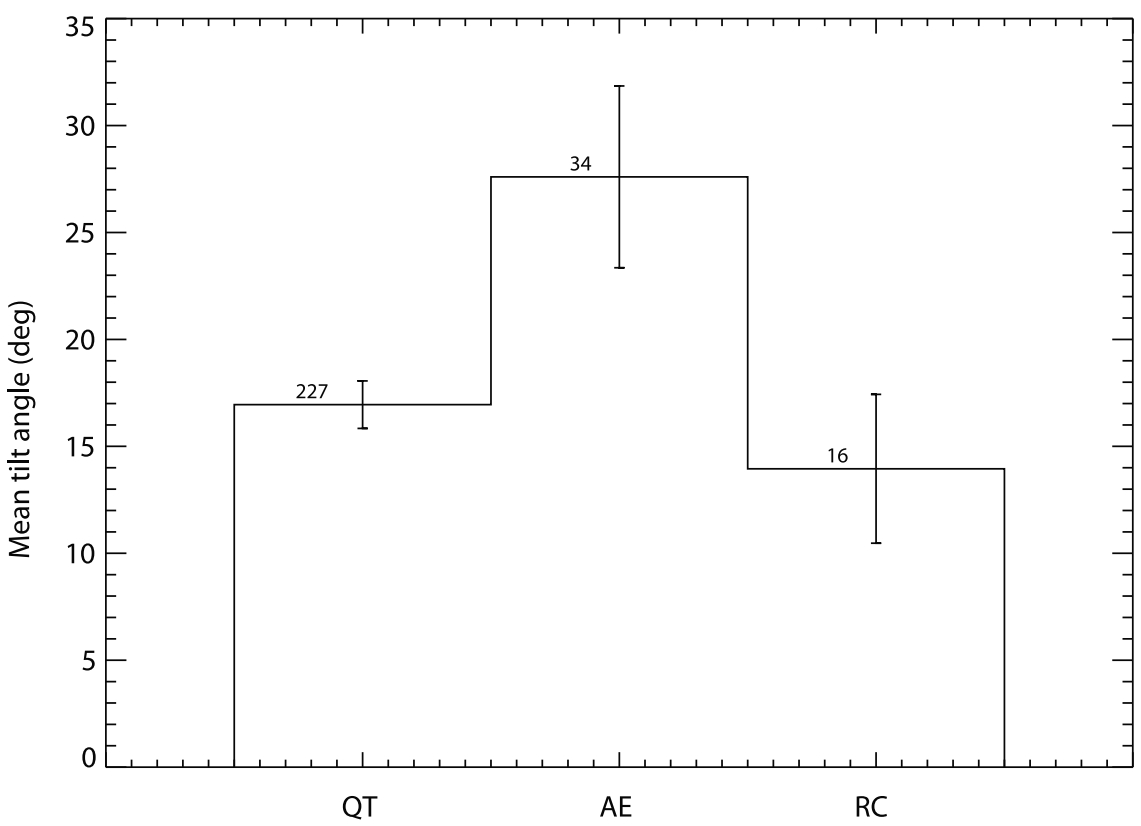

Figure 5. Mean tilt angle for different geomagnetic conditions for 2001-2007 data. 'QT' represent those crossings where SYM-H $>-50 \mathrm{nT}$ and $\mathrm{AE}<500 \mathrm{nT}$. The 'AE' data include all crossings where SYM-H $>$ $-50 \mathrm{nT}$ and $\mathrm{AE} \geq 500 \mathrm{nT}$. The 'RC' group are crossings where $\mathrm{SYM}-\mathrm{H} \leq-50 \mathrm{nT}$ and $\mathrm{AE} \geq 500 \mathrm{nT}$. Standard errors in the means are shown by vertical lines through each data point and the numbers in each group are shown above each bar.

and 2002, the mean SYM-H values for the 'RC' group are more negative, indicating a more enhanced ring current, compared to years 2003 and 2004. In addition, the 'QT' groups in each year show a variation in magnitude of the maximum current density even though the mean $\mathrm{AE}$ and SYM-H values for the QT groups are fairly similar in each year. This effect may be due to the spacecraft separation, with

Mean SYM-H $(n T)$
$\mathrm{QT}=-10.3$
$\mathrm{AE}=-36.1$
$\mathrm{RC}=-101.6$
Mean AE $(\mathrm{nT})$
$\mathrm{QT}=208.4$
$\mathrm{AE}=959.5$
$\mathrm{RC}=755.2$

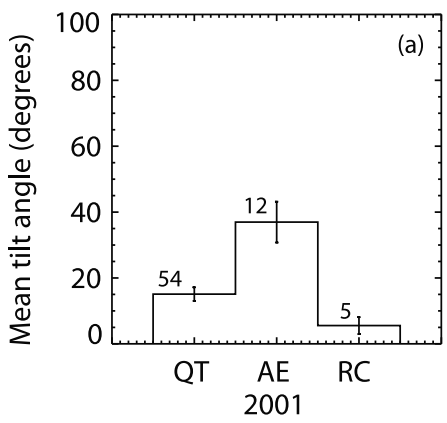

Mean SYM-H (nT)

$\mathrm{QT}=-5.3$

$A E=-45.0$

$R C=-60.0$

Mean AE (nT)

$\mathrm{QT}=222.5$

$\mathrm{AE}=926.7$

$\mathrm{RC}=1400.8$

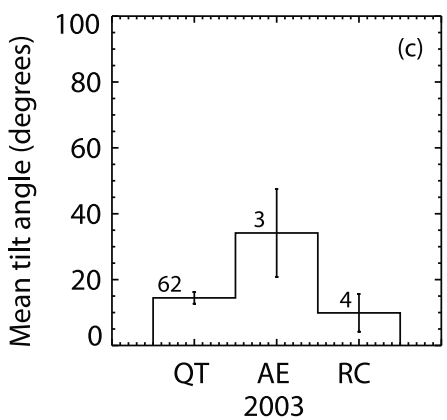

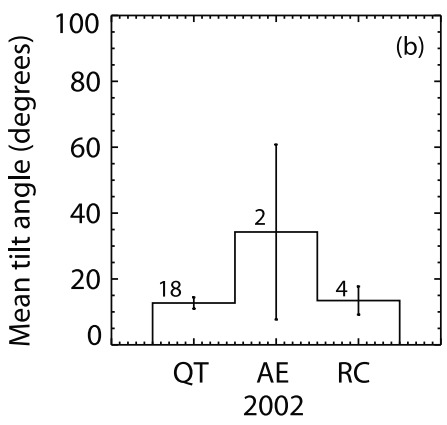
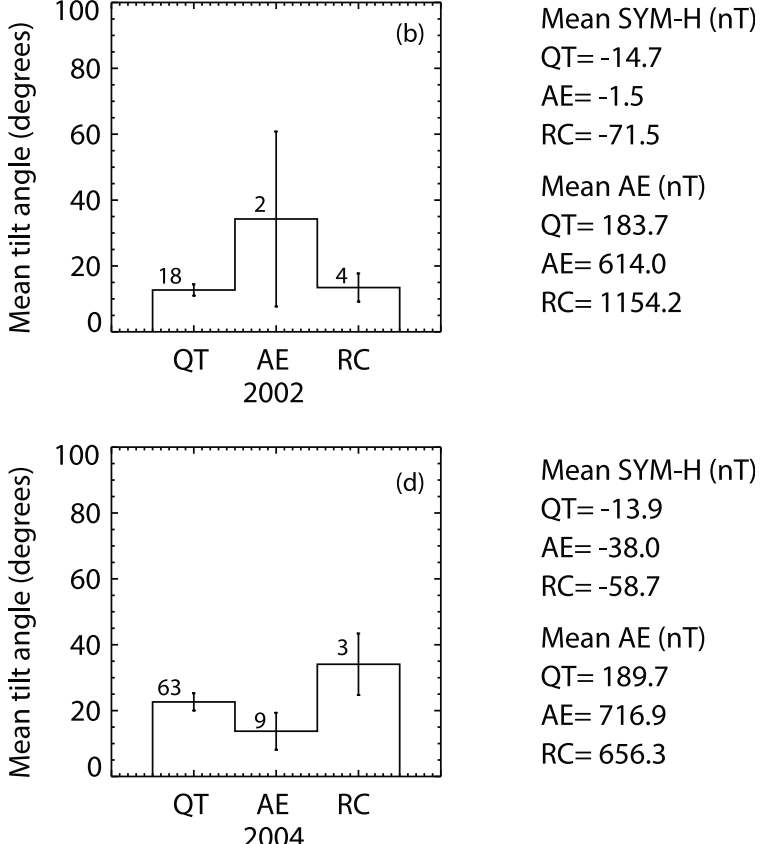

Mean SYM-H (nT)

$\mathrm{QT}=-13.9$

$A E=-38.0$

$\mathrm{RC}=-58.7$

Mean AE (nT)

$\mathrm{QT}=189.7$

$\mathrm{AE}=716.9$

$\mathrm{RC}=656.3$

Figure 6. (a-d) The mean tilt angle for different geomagnetic conditions for 2001-2004 only. For each panel, 'QT' represents those crossings where SYM-H $>-50 \mathrm{nT}$ and $\mathrm{AE}<500 \mathrm{nT}$. 'AE' includes all crossings where SYM-H $>-50 \mathrm{nT}$ and $\mathrm{AE} \geq 500 \mathrm{nT}$. 'RC' is a group of crossings where SYM-H $\leq-50 \mathrm{nT}$ and $\mathrm{AE} \geq 500 \mathrm{nT}$. Standard errors in the means are shown by vertical lines through each bar and the numbers within each group are shown above each bar. 

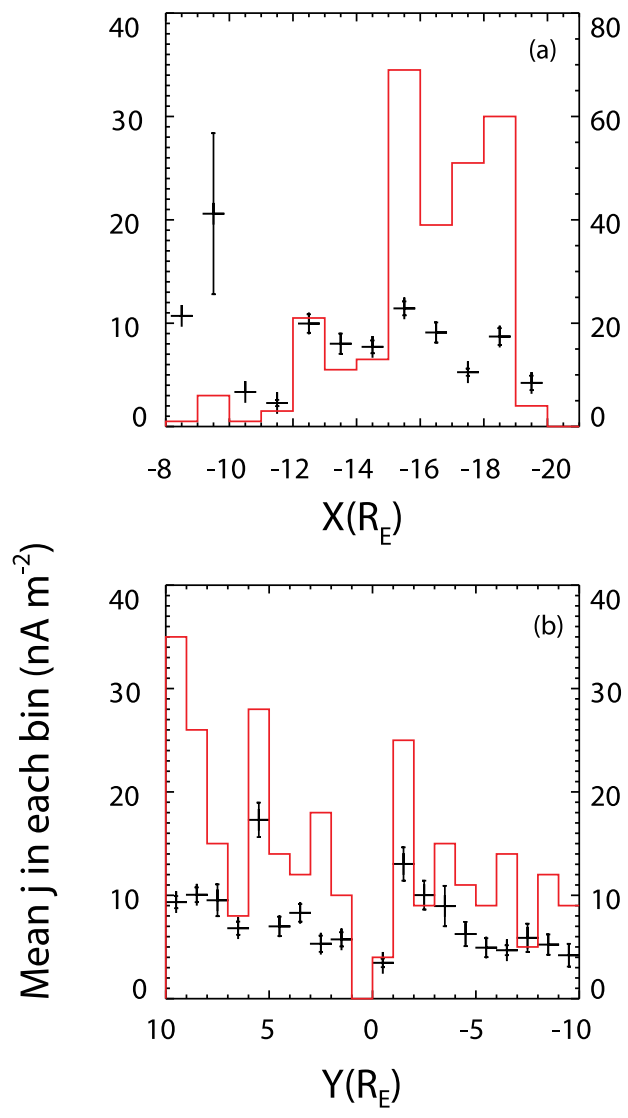

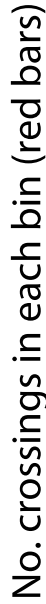

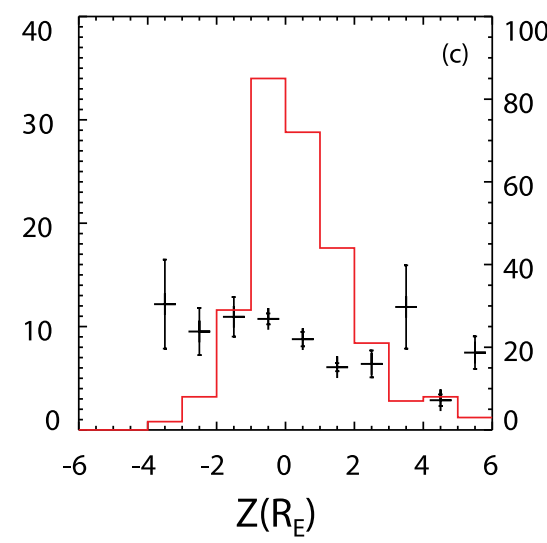

Figure 7. The means of the maximum current density magnitude reached in each crossing (using the left hand $\mathrm{Y}$ axis) for 2001-2007 data, in 1 $\mathrm{R}_{E}$ bins of the starting position of the crossings in (a) $\mathrm{X}$, (b) $\mathrm{Y}$ and (c) Z (GSM coordinates). Standard errors in the means are shown by vertical lines through each data point. The right hand side axis shows the numbers in each bin, indicated by the red bars.

larger current densities found in 2003 at the smallest separation $(200 \mathrm{~km})$ and smaller values at larger separations.

\section{Discussion}

[19] Our investigation focused on the cross-tail current sheet and studied its orientation in the YZ plane and current density. We used data from Cluster's 2001-2007 tail seasons to examine whether the orientation and current density of the current sheet varied with three different levels of geomagnetic activity, defined using the AE and SYM-H indices. Our study has shown that the tilt of the current sheet is larger during times of increased substorm activity and that when the ring current is enhanced the tilt angle is reduced. We have also shown that the current density varies with substorm and storm activity, with larger current densities observed during magnetic storms when the ring current is enhanced.

[20] There was some indication from Figures 2 and 3 that there was a smaller tilt angle in crossings where the ring current was enhanced, implying that during magnetic storms the current sheet may be stabilized in terms of its orientation. The relationship with the AE index shown in Figure 3 implied that crossings of the current sheet during non-storm times had larger tilt angles than those during storm conditions and that the tilt angle increased with increasing $\mathrm{AE}$, although the number of data points above $500 \mathrm{nT}$ were fewer than where the $\mathrm{AE}<500 \mathrm{nT}$. Figure 4 showed that the tilt angle also varied with position of the crossing. It suggested that the tilt angle may be slightly larger in the dawn flank (Y GSM direction) and at larger values of $Z(G S M)$. The observation at high $Z$ values is to be expected as the Cluster tail season occurs when the neutral sheet is located at positive $Z$, due to the tilt of the Earth. Tsyganenko et al. [1998] showed that the magnetotail current sheet is tilted away from the XY plane, with this warping increasing toward the flanks. As such, we would expect to see higher tilt angles at the flanks of the magnetosphere as the current sheet is naturally tilted away from the XY plane at these locations. The effect of the IMF $\mathrm{B}_{Y}$ on the tilt in the $\mathrm{YZ}$ plane was shown to be larger with increasing distance downtail as Tsyganenko et al. [1998] considered distances up to $100 \mathrm{R}_{E}$ downtail. We have not considered the effect of IMF $\mathrm{B}_{Y}$ in our study, although based on the data from Tsyganenko et al. [1998], we estimate any effect to be between $6-9^{\circ}$, for IMF $\mathrm{B}_{Y}=10 \mathrm{nT}$, in both flanks at the downtail distances considered in our study.

[21] The overall picture of the data (Figure 5), comparing geomagnetic conditions for 2001-2007, clearly demonstrated the increased tilt angle during high values of the $\mathrm{AE}$ index and lower tilt angles during quiet and storm times. The first four years of data were then considered (Figure 6) and for 2001-2003 the groups of crossings with the enhanced ring current $(\mathrm{RC})$ had lower tilt angles than the groups with the large $\mathrm{AE}$ indices and a quiet ring current (AE), although there was large variation for the $\mathrm{AE}$ group in 2002 and 2003. In contrast, 2004 did not follow this pattern. However, when the mean values of SYM-H for the enhanced ring current groups ( $\mathrm{RC}$ ) were compared, it was clear that the mean value of SYM-H for 2004 was more positive than in the previous years. The results suggest that crossings occurring when there is an enhanced ring current have a lower current sheet tilt angle, especially with increasing strength of the ring current.

[22] The range of tilt angles calculated in this study are in agreement with those observed by others such as Zhang et al. [2002], Runov et al. [2005b] and Sergeev et al. [2003, 2004]. The larger tilt angles noted by others were generally found in flapping current sheets. Although this study did not investigate current sheet flapping, a previous study [Davey et al., 


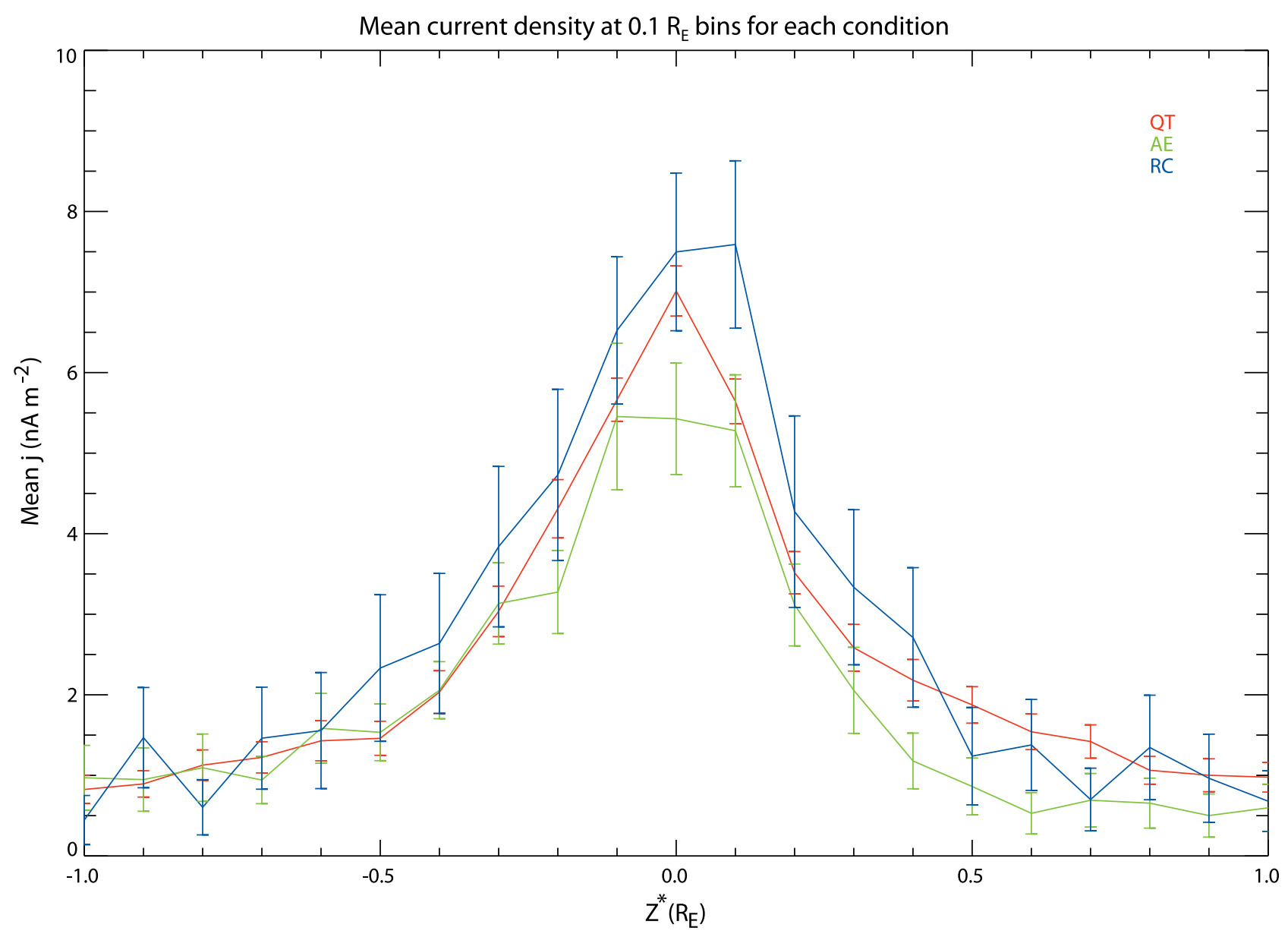

Figure 8. Mean current density magnitude for different geomagnetic conditions for 2001-2007 data, in $0.1 \mathrm{R}_{E}$ bins of $Z^{*}$. The red data $(\mathrm{QT})$ represent those crossings where SYM-H $>-50 \mathrm{nT}$ and $\mathrm{AE}<$ $500 \mathrm{nT}$. The green data (AE) include all crossings where SYM-H $>-50 \mathrm{nT}$ and AE $\geq 500 \mathrm{nT}$. The blue data $(\mathrm{RC})$ are crossings where $\mathrm{SYM}-\mathrm{H} \leq-50 \mathrm{nT}$ and $\mathrm{AE} \geq 500 \mathrm{nT}$. Standard errors in the means are shown by vertical lines through each data point.

2012], found that current sheet flapping was associated with enhanced substorm conditions. The present study found that increased substorm conditions are associated with larger tilt angles and as such the combination of the present study and Davey et al. [2012] are in agreement with the previous studies. In addition Davey et al. [2012] found that during magnetic storms, the flapping motion was generally decreased. Other work [Petrukovich et al., 2005] showed lower tilt angles for an inactive current sheet. The present study, showing that during magnetic storms there are on average lower tilt angles, is again in agreement with what might be surmised on combining studies such as Petrukovich et al. [2005] and Davey et al. [2012].

[23] The current density analysis also separated the data into geomagnetic condition groups and Figures 8 and 9 both showed that the groups of crossings with an enhanced ring current (RC) had higher maximum current densities compared to the other groups, specifically the group with high values of the $\mathrm{AE}$ index and a quiet ring current (AE). Research by, for example, Runov et al. [2003, 2006] and Sergeev et al. [2003], has shown a bifurcated structure of the current sheet. Runov et al. [2006] studied 30 rapid current sheet crossings using 2001 Cluster data and found that 5 out of those were bifurcated. They found no AE-dependence on the different types of thickness of current sheet. Our study did not restrict the duration of crossings and as such we cannot estimate from the Runov et al. [2006] data, how many of the crossings in our study would be classified as bifurcated in structure. It is possible that bifurcation did occur in a proportion of the crossings and as such any averaging may have reduced the current density at $Z^{*}=0$. However, we cannot assume that this would have occurred in one group more than the other groups and as such have not included any corrections for possible bifurcation. The lower current density for the 'AE' group may be due to the diversion of the tail current into the ionosphere during the onset of the expansion phase of a substorm, although we have not separated the data according to the substorm phase and so this has not been assessed. Figure 8 also shows a slightly wider current profile for the ' $\mathrm{RC}$ ' group compared to the other geomagnetic condition groups, implying a larger half thickness. Estimates of half thickness taken from Figure 8 are approximately $0.4 \mathrm{R}_{E}(\approx 2500 \mathrm{~km})$ for the ' $\mathrm{RC}$ ' group, $0.26 \mathrm{R}_{E}(\approx 1700 \mathrm{~km})$ for the 'QT' group and $\approx 0.24 \mathrm{R}_{E}(\approx 1500 \mathrm{~km})$ for the 'AE' group. These are values taken from average current density estimates for groups of crossings. They are however in 
(a)

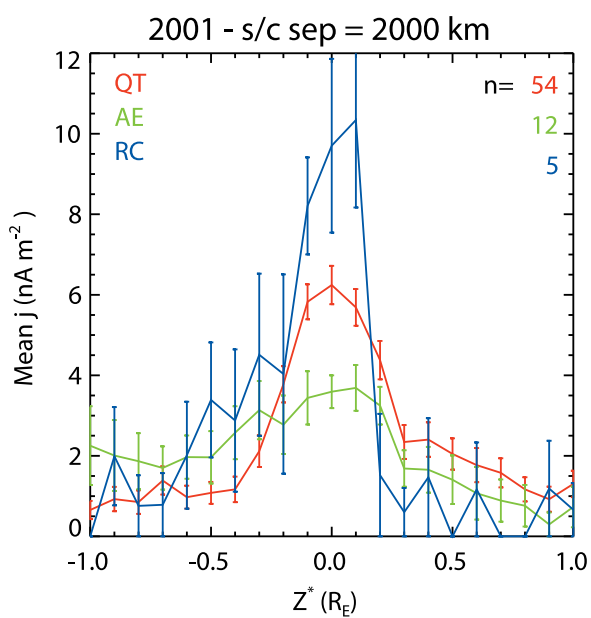

(c)

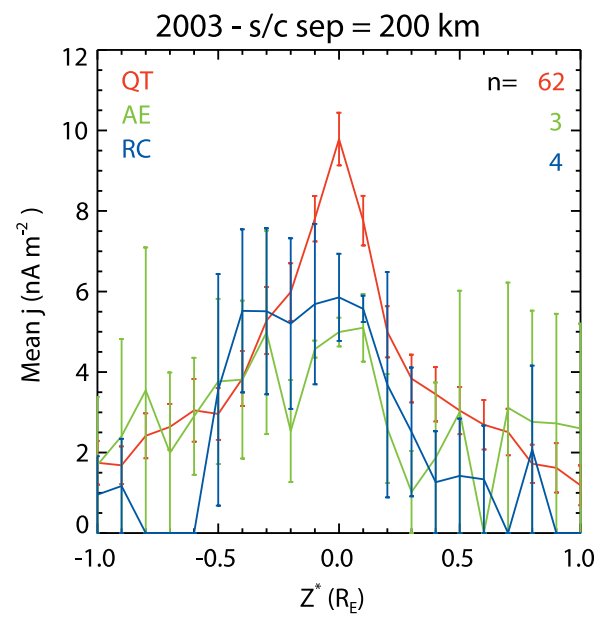

(b)
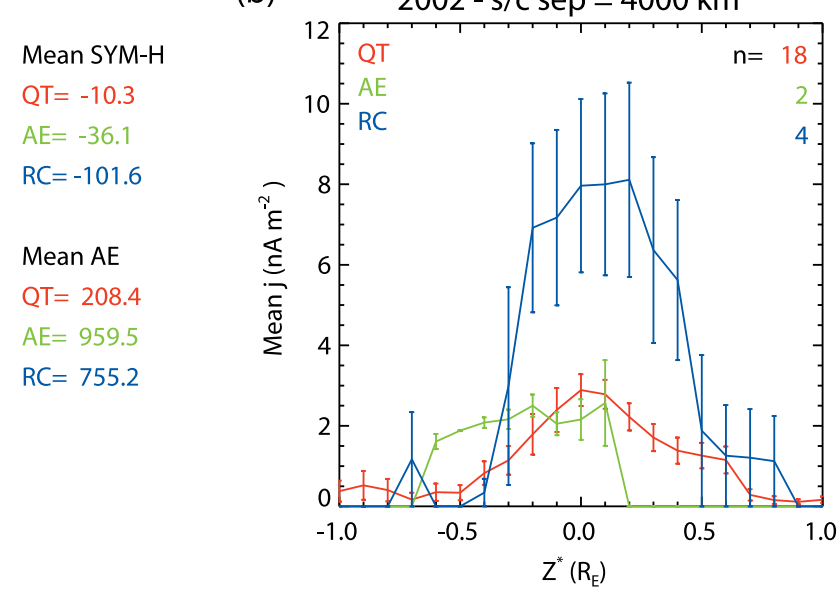

Mean SYM-H

$\mathrm{QT}=-14.7$

$\mathrm{AE}=-1.5$

$\mathrm{RC}=-71.5$

Mean AE

$\mathrm{QT}=183.7$

$\mathrm{AE}=614.0$

$\mathrm{RC}=1154.2$

(d)

Mean SYM-H

$\mathrm{QT}=-5.3$

$A E=-45.0$

$R C=-60.0$

Mean AE

$\mathrm{QT}=222.5$

$A E=926.7$

$R C=1400.8$

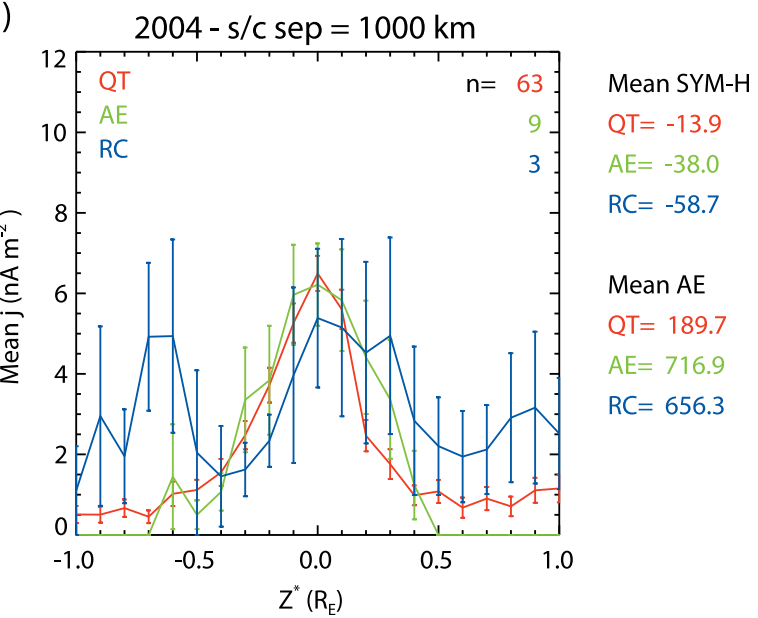

Figure 9. (a-d) Mean current density magnitude for different geomagnetic conditions for 2001-2004 data, in $0.1 \mathrm{R}_{E}$ bins of $\mathrm{Z}^{*}$. The red data $(\mathrm{QT})$ represent those crossings where SYM-H $>-50 \mathrm{nT}$ and $\mathrm{AE}<500 \mathrm{nT}$. The green data (AE) include all crossings where SYM-H $>-50 \mathrm{nT}$ and $\mathrm{AE} \geq 500 \mathrm{nT}$. The blue data (RC) are crossings where SYM-H $\leq-50 \mathrm{nT}$ and $\mathrm{AE} \geq 500 \mathrm{nT}$. Standard errors in the means are shown by vertical lines through each data point. The numbers in each group are shown in the top right hand corner of each plot.

agreement with previous estimations, such as Sergeev et al. [2003] who found a half thickness of $0.36 \mathrm{R}_{E}$ for a current sheet flapping event in 2001 and Rong et al. [2011]. Runov et al. [2005a] cited half thickness values of $\leq 1000 \mathrm{~km}$ for the majority of fast current sheet crossing events studied. A larger range of values was calculated by Runov et al. [2005b] (1500-10,000 km) for a statistical study of 78 rapid current sheet crossings, and later Runov et al. [2006] found typical values of $<2000 \mathrm{~km}$ and $4000 \mathrm{~km}$ (for bifurcated current sheets) for fast crossings during Cluster's 2001 tail season.

[24] If the averaged estimates of half thickness are considered to be accurate then spacecraft separations of 1000 $2000 \mathrm{~km}$ should be adequate to resolve the current densities for the crossings and as such 2001 and 2004 data in Figure 9 should show good estimates of the current density profiles for each group. In contrast, the values in 2002 at $4000 \mathrm{~km}$ separation may have underestimated the values somewhat for all groups. Forsyth et al. [2011] noted that the curlometer technique underestimates the current density, although it improves for larger current widths. They also concluded that for currents that are about half the width of the spacecraft separation, the curlometer detects about $80 \%$ of the current. In addition, Runov et al. [2005b] calculated that the current density magnitude could be underestimated up to $30 \%$ and the half thickness up to $15 \%$. The 'QT' groups in 2001 and 2004 show similar profiles. The 'AE' group has larger maximum current density in 2004 , with a slightly lower mean value of the $\mathrm{AE}$ index than in 2001. However, the ' $\mathrm{RC}$ ' group has a larger maximum current density in 2001, where the mean value of SYM-H for the group is more negative than in 2004, indicating a possible effect of the strength of the ring current on the current density. In general, larger current densities are seen in the ' $\mathrm{RC}$ ' group for years 2001 and 2002 where the mean SYM-H values are less than $-70 \mathrm{nT}$. 
However, in the later years, the mean current density values of the 'RC' groups are very similar to the 'AE' groups and here the mean SYM-H values are $-59 \mathrm{nT}(2003)$ and $-60 \mathrm{nT}$ (2004) for the 'RC' groups. The results demonstrate that as with the tilt angle, the strength of the ring current may affect the magnetic configuration of the magnetosphere such that the current density within the current sheet during a crossing is higher when SYM-H is less than about $-70 \mathrm{nT}$, implying moderate storm activity.

[25] The current density values estimated in this paper are also in agreement with those noted in previous work such as Rong et al. [2011], Runov et al. [2005b, 2006], Shen et al. [2008], Sergeev et al. [2003], and Petrukovich et al. [2007], with observations of maximum current density of less than $10 \mathrm{nA} \mathrm{m}^{-2}$ as well as values over $30 \mathrm{nA} \mathrm{m}^{-2}$ for individual crossings. The mean values in specific groups have been presented for the purposes of this paper, rather than individual crossings.

[26] Previous work by Milan et al. [2008] showed that the magnetotail contains more open flux during magnetic storms, suggesting that it becomes stabilized to substorm initiation at these times and that more open flux is needed for reconnection to occur. Davey et al. [2012] found that when the ring current is enhanced the current sheet is stabilized in terms of its motion. They also found that there is more motion when the standard deviation of the AE index implies stronger substorm activity and a quiet ring current. The results from the present study suggest that the enhanced ring current also has an effect of reducing the tilt angle of the current sheet, when SYM-H values are approaching $-100 \mathrm{nT}$. In addition, our results show an increased tilt angle of the current sheet during times when the $\mathrm{AE}$ index is $\geq 500 \mathrm{nT}$, implying strong substorm activity. Further, our results show larger current densities during storm times compared to quiet and substorm times.

\section{Conclusions}

[27] An investigation was carried out of the orientation and current density within the cross-tail current sheet for current sheet crossings between 2001 and 2007, using the Cluster spacecraft.

[28] Our results suggest that crossings occurring when the $\mathrm{AE}$ index is large $(>500 \mathrm{nT})$ with a quiet ring current involve larger values of tilt angle and a reduced current density during crossings. In contrast, crossings during substorms but with an enhanced ring current (during magnetic storms) involve a reduced tilt angle and an increased current density, with the strength of the enhanced ring current impacting on how much the current density and tilt angle are affected. We propose that the increased amount of flux in the lobe during magnetic storms, suggested by Milan et al. [2008], causes an increased pressure on the current sheet, causing it to not only have a reduced motion as found by Davey et al. [2012], but also a smaller tilt angle in the $\mathrm{YZ}$ plane and a larger current density caused by the larger change in $\mathrm{B}_{X}$ in the lobes. The effect of interplanetary conditions have not been investigated here, although the work could be extended in the future to examine this. In addition, any disturbances such as fast flows and bifurcations of the current sheet have not been studied and again these would be important areas to focus on for the future. Further work will also investigate how the lobe field changes during these crossings and how different geomagnetic conditions may affect its configuration.

[29] Acknowledgments. We wish to thank the Cluster Active Archive (CAA) for the spin resolution FGM data and the CIS data and the Kyoto World Data Centre for Geomagnetism for the SYM-H and AE data. We also thank the operations teams for the instruments used in the study. M. Lester, S. E. Milan and R. C. Fear are supported by STFC grant ST/H002480/1. E. A. Davey is supported by a STFC studentship (X89ST17). C. Forsyth is supported by STFC grant ST/H00260X/1.

[30] Masaki Fujimoto thanks the reviewers for their assistance in evaluating this paper.

\section{References}

Balogh, A., et al. (2001), The Cluster Magnetic Field Investigation: Overview of in-flight performance and initial results, Ann. Geophys., 19 , 1207-1217, doi:10.5194/angeo-19-1207-2001.

Barlow, R. J. (1989), Statistics: A Guide to the Use of Statistical Methods in the Physical Sciences, John Wiley, Chichester, U. K.

Dandouras, I., A. Barthe, E. Penou, S. Brunato, H. Rème, L. M. Kistler, M. B. Bavassano-Cattaneo, and A. Blagau (2010), Cluster Ion Spectrometry (CIS) data in the Cluster Active Archive (CAA), in The Cluster Active Archive, Studying the Earth's Space Plasma Environment, edited by H. Laakso, M. Taylor, and C. P. Escoubet, pp. 51-72, Springer, Dordrecht, Netherlands.

Davey, E. A., M. Lester, S. E. Milan, and R. C. Fear (2012), Storm and substorm effects on magnetotail current sheet motion, J. Geophys. Res., 117 A02202, doi:10.1029/2011JA017112.

Davis, T. N., and M. Sugiura (1966), Auroral electrojet activity index AE and its universal time variations, J. Geophys. Res., 71, 785-801.

Dunlop, M. W., D. J. Southwood, K.-H. Glassmeier, and F. M. Neubauer (1988), Analysis of multipoint magnetometer data, Adv. Space Res., 8, 273-277, doi:10.1016/0273-1177(88)90141-X.

Forsyth, C., M. Lester, R. C. Fear, E. Lucek, I. Dandouras, A. N. Fazakerley, H. Singer, and T. K. Yeoman (2009), Solar wind and substorm excitation of the wavy current sheet, Ann. Geophys., 27, 2457-2474, doi:10.5194/ angeo-27-2457-2009.

Forsyth, C., M. Lester, A. N. Fazakerley, C. J. Owen, and A. P. Walsh (2011), On the effect of line current width and relative position on the multi-spacecraft curlometer technique, Planet. Space Sci., 59, 598-605, doi:10.1016/j.pss.2009.12.007.

Gloag, J. M., E. A. Lucek, L. Alconcel, A. Balogh, P. Brown, C. M. Carr, C. N. Dunford, T. Oddy, and J. Soucek (2010), FGM data products in the CAA, in The Cluster Active Archive, Studying the Earth's Space Plasma Environment, edited by H. Laakso, M. Taylor, and C. P. Escoubet, pp. 109-128, Springer, Dordrecht, Netherlands.

Iyemori, T. (1990), Storm-time magnetospheric currents inferred from mid-latitude geomagnetic field variations, J. Geomagn. Geoelectr., 42, 1249-1265.

Laakso, H., C. Perry, S. McCaffrey, D. Herment, A. J. Allen, C. C. Harvey, C. P. Escoubet, C. Gruenberger, M. G. G. T. Taylor, and R. Turner (2010), Cluster Active Archive: Overview, in The Cluster Active Archive, Studying the Earth's Space Plasma Environment, edited by H. Laakso, M. Taylor, and C. P. Escoubet, pp. 3-37, Springer, Dordrecht, Netherlands.

Milan, S. E., P. D. Boakes, and B. Hubert (2008), Response of the expanding/ contracting polar cap to weak and strong solar wind driving: Implications for substorm onset, J. Geophys. Res., 113, A09215, doi:10.1029/ 2008JA013340.

Nakamura, R., W. Baumjohann, Y. Asano, A. Runov, A. Balogh, C. J. Owen, A. N. Fazakerley, M. Fujimoto, B. Klecker, and H. Rème (2006), Dynamics of thin current sheets associated with magnetotail reconnection, J. Geophys. Res., 111, A11206, doi:10.1029/2006JA011706.

Perry, C., T. Eriksson, P. Escoubet, S. Esson, H. Laakso, S. McCaffrey, T. Sanderson, H. Bowen, A. Allen, and C. Harvey (2006), The ESA Cluster Active Archive, in Cluster and Double Star Symposium, ESA Spec. Publ., SP-598.

Petrukovich, A. A., W. Baumjohann, R. Nakamura, A. Runov, and A. Balogh (2005), Cluster vision of the magnetotail current sheet on a macroscale, J. Geophys. Res., 110, A06204, doi:10.1029/2004JA010825. Petrukovich, A. A., W. Baumjohann, R. Nakamura, A. Runov, A. Balogh, and H. Rème (2007), Thinning and stretching of the plasma sheet, J. Geophys. Res., 112, A10213, doi:10.1029/2007JA012349.

Rème, H., et al. (2001), First multispacecraft ion measurements in and near the Earth's magnetosphere with the identical Cluster Ion Spectrometry (CIS) experiment, Ann. Geophys., 19, 1303-1354, doi:10.5194/angeo19-1303-2001.

Robert, P., M. W. Dunlop, A. Roux, and G. Chanteur (1998), Accuracy of current density determination, ISSI Sci. Rep. Ser., 1, 395-418. 
Rong, Z., C. Shen, E. Lucek, A. Balogh, and L. Yao (2010), Statistical survey on the magnetic field in magnetotail current sheets: Cluster observations, Chin. Sci. Bull., 55(23), 2542-2547, doi:10.1007/s11434-0103096-5.

Rong, Z. J., W. X. Wan, C. Shen, X. Li, M. W. Dunlop, A. A. Petrukovich, T. L. Zhang, and E. Lucek (2011), Statistical survey on the magnetic structure in magnetotail current sheets, J. Geophys. Res., 116, A09218, doi:10.1029/2011JA016489.

Runov, A., R. Nakamura, W. Baumjohann, T. L. Zhang, M. Volwerk, H. Eichelberger, and A. Balogh (2003), Cluster observation of a bifurcated current sheet, Geophys. Res. Lett., 30(2), 1036, doi:10.1029/ 2002 GL016136.

Runov, A., V. A. Sergeev, R. Nakamura, W. Baumjohann, T. L. Zhang, Y. Asano, M. Volwerk, Z. Vörös, A. Balogh, and H. Rème (2005a), Reconstruction of the magnetotail current sheet structure using multi-poin Cluster measurements, Planet. Space Sci., 53, 237-243, doi:10.1016/j. pss.2004.09.049.

Runov, A., et al. (2005b), Electric current and magnetic field geometry in flapping magnetotail current sheets, Ann. Geophys., 23, 1391-1403, doi:10.5194/angeo-23-1391-2005b.

Runov, A., et al. (2006), Local structure of the magnetotail current sheet: 2001 Cluster observations, Ann. Geophys., 24, 247-262, doi:10.5194/ angeo-24-247-2006.

Sergeev, V., et al. (2003), Current sheet flapping motion and structure observed by Cluster, Geophys. Res. Lett., 30(6), 1327, doi:10.1029/ 2002GL016500.
Sergeev, V., A. Runov, W. Baumjohann, R. Nakamura, T. L. Zhang, A. Balogh, P. Louarnd, J. Sauvaud, and H. Reme (2004), Orientation and propagation of current sheet oscillations, Geophys. Res. Lett., 31 , L05807, doi:10.1029/2003GL019346.

Sergeev, V. A., D. A. Sormakov, S. V. Apatenkov, W. Baumjohann, R. Nakamura, A. V. Runov, T. Mukai, and T. Nagai (2006), Survey of large-amplitude flapping motions in the midtail current sheet, Ann. Geophys., 24, 2015-2024, doi:10.5194/angeo-24-2015-2006.

Shen, C., Z. J. Rong, X. Li, M. Dunlop, Z. X. Liu, H. V. Malova, E. Lucek, and C. Carr (2008), Magnetic configurations of the tilted current sheets in magnetotail, Ann. Geophys., 26, 3525-3543, doi:10.5194/angeo-26-35252008.

Sonnerup, B. U. Ö., and M. Scheible (1998), Minimum and maximum variance analysis, ISSI Sci. Rep. Ser., 1, 185-220.

Tsyganenko, N. A., S. B. P. Karlsson, S. Kokubun, T. Yamamoto, A. J. Lazarus, K. W. Ogilvie, C. T. Russell, and J. A. Slavin (1998), Global configuration of the magnetotail current sheet as derived from Geotail, Wind, IMP 8 and ISEE 1/2 data, J. Geophys. Res., 103, 6827-6842, doi:10.1029/97JA03621.

Zhang, T. L., W. Baumjohann, R. Nakamura, A. Balogh, and K. Glassmeier (2002), A wavy twisted neutral sheet observed by CLUSTER, Geophys. Res. Lett., 29(19), 1899, doi:10.1029/2002GL015544.

Zhang, T. L., et al. (2006), A statistical survey of the magnetotail current sheet, Adv. Space Res., 38, 1834-1837, doi:10.1016/j.asr.2006.05.009. 